Y. SHIBUYA

KODAI MATH. J.

1 (1978), 197-204

\title{
ON THE EXISTENCE OF A COMPLEX ALMOST CONTACT STRUCTURE
}

\author{
By Yhuji Shibuya
}

\section{$\S 1$. Introduction.}

A complex manifold of complex dimension $2 m+1$ is said to be a complex contact manifold if it admits an open covering $\left\{\tilde{O}_{i}\right\}$ such that on each $\tilde{O}_{\imath}$ there is a holomorphic 1-form $\gamma_{2}$ with $\gamma_{2} \wedge\left(d \gamma_{2}\right)^{m} \neq 0$ and on $\tilde{O}_{\imath} \cap \tilde{O}_{\jmath} \neq \phi, \quad \gamma_{i}=\tilde{f}_{2}, \gamma_{3}$, for some non-vanishing holomorphic function $\tilde{f}_{\imath \jmath}$. In general such a structure is not given by a global 1-form $\gamma$; in fact, this is the case for a complex manifold if and only if its first Chern class vanishes [7]. It is also shown in [7] that the structural group of the tangent bundle of a complex contact manifold is reducible to $(S p(m) \otimes U(1)) \times U(1)$. Standard examples of complex contact manifolds are the odd dimensional complex projective space $P C^{2 m+1}$, the complex projective cotangent bundle of a complex manifold, etc.. (See [3], [7].)

On the other hand, some of complex contact manifolds are base spaces of principal fibre bundles with 1-dimensional fibres and real contact 3-structure. A typical example of this is a Hopf map $S^{4 m+3} \longrightarrow P C^{2 m+1}$. Generalizing this situation Ishihara and Konishi studied in [5] fiberings with 1-dimensional fibres of a manifold with real contact 3-structure and defined in the base space a new structure called a complex almost contact structure. In [2] an equivalent definition is given in terms of global tensor fields. The structural group of the tangent bundle of a complex almost contact manifold is also reducible to $(S p(m) \otimes U(1)) \times U(1)$. The notion of a complex almost contact structure is naturally weaker than that of a complex contact structure. In fact, a manifold with a complex contact structure admits a complex almost contact structure, and the converse is true if the complex almost contact structure is normal $[5],[6]$.

Let $M$ be a complex manifold of complex dimension $2 m+1$. Let $\mathcal{O}=\left\{O_{i}\right\}$ be an open covering of $M$. We say, in this paper, $M$ has a $\Omega$-structure if the structural group of the tangent bundle of $M$ is reducible to $(S p(m) \otimes U(1)) \times U(1)$, that is equivalent to the existence of a local 2 -form $\Omega_{\imath}$ of type $(2,0)$ on each $O_{\imath}$ such that $\left(\Omega_{\imath}\right)^{m} \neq 0$ and a non-vanishing function $f_{\imath j} \in U(1)$ such that $\Omega_{\imath}=f_{\imath}, \Omega_{\text {, }}$ on $O_{\imath} \cap O_{j} \neq \phi$.

Received February 22, 1977. 
The existence of a real almost contact structure is discussed by Hatakeyama [4]. In this paper we generalize his result to the complex case and prove that the existence of a $\Omega$-structure is a sufficient condition for the existence of a complex almost contact structure.

\section{§ 2. Decomposition of $G L(n, C)$.}

Let $H^{+}(n)$ denote the set of all positive definite hermitian ( $\left.n, n\right)$-matrices. Then $H^{+}(n)$ is naturally imbedded as a real analytic submanifold in the general linear group $\mathrm{GL}(n, C)$, and its analytic structure is defined by the components of its element. Let $U(n)$ denote the unitary group with usual analytic structure. Both $H^{+}(n)$ and $U(n)$ are considered to act on $n$-complex variables. Algebraically it is well known that any $\lambda \in \mathrm{GL}(n, C)$ is written in one and only one way as the product $\lambda=\eta v$ of $\eta \in H^{+}(n)$ and $v \in U(n)$, thus the components of $\eta$ and $v$ of the decomposition of $\lambda$ depend continuously on those of $\lambda$. In this section we check this decomposition is in fact analytic. First we prove

Proposition 1. Let $\Phi$ be a map from the product manifold $H^{+}(n) \times U(n)$ to the group $\mathrm{GL}(n, \boldsymbol{C})$ defined by

$$
\Phi(\eta, v)=\eta v, \quad \eta \in H^{+}(n), \quad v \in U(n) .
$$

Then $\Phi$ is an analytıc map, and its differential map $d \Phi$ is everywhere an onto ısomorphism.

In order to prove Proposition 1 we begin with the following

LEMMA 1. Let $\eta$ be an element of $H^{+}(n)$. Then the characteristic values of the linear map ad $(\eta): \mathfrak{g l}(n, C) \longrightarrow \mathfrak{g l}(n, C)$, defined by

$$
\operatorname{ad}(\eta) L=\eta L \eta^{-1}, \quad L \in \mathfrak{g l}(n, \boldsymbol{C}),
$$

are all positive numbers.

Proof. Since $\eta$ belongs to $H^{+}(n)$, the characteristic values of $\eta$ are all positive real numbers, which we shall denote by $a_{\imath}(i=1, \cdots, n)$. According to a classical result, there is a unitary matrix $\nu$ such that $\nu \eta \nu^{-1}$ is a diagonal matrix $\delta$ whose diagonal components are the $a_{\imath}$ 's. Since $a d(\eta)=a d(\nu)^{-1} a d(\delta) a d(\nu)$ the characteristic values of $a d(\eta)$ are equal to those of $a d(\delta)$ and hence, by direct computation, equal to the $n^{2}$ real numbers $a_{\imath} a_{\jmath}^{-1}(\imath, \jmath=1, \cdots, n)$, which are all positive real numbers.

q. e. d.

COROllary. Let $A$ be a skew hermitian $(n, n)$-matrix and $\eta$ be an element of $H^{+}(n)$. If $\eta A$ is a hermitian matrix, then $A=0$. 
Proof. If $\eta A$ is a hermitian matrix, then

$$
\eta A={ }^{t} \overline{\eta A}={ }^{t} \bar{A}^{t} \bar{\eta}=-A \eta,
$$

that is $\operatorname{ad}(\eta) A=-A$. Thus $A=0$ by Lemma 1 .

q. e. d.

Proof of Proposition 1. $\Phi$ is an analytic map because $H^{+}(n)$ and $U(n)$ are real analytic submanifolds of $\mathrm{GL}(n, C)$ and the multiplication of the elements of $\mathrm{GL}(n, \boldsymbol{C})$ is algebraic. In order to prove $d \Phi$ is everywhere an onto isomorphism we have only to prove that the differential map $d \Phi$ is univalent since the dimensions of $H^{+}(n) \times U(n)$ and $\operatorname{GL}(n, C)$ are both equal to $2 n^{2}$. Let $X$ be a tangent vector to $H^{+}(n) \times U(n)$ at a point $(\eta, v)$ which is tangent to a parameterized curve $(\eta+t B, v \exp t A)$, where $A$ is skew hermitian and $B$ is hermitian. Then $d \Phi(X)$ is given by

$$
\begin{aligned}
d \Phi(X) & =\lim _{t \rightarrow 0} \frac{(\eta+t B)(v \exp t A)-\eta v}{t} \\
& =\eta A v+B v .
\end{aligned}
$$

Assuming $d \Phi(X)=0$, we have $\eta A v+B v=0$ and therefore $\eta A=-B$, which means $\eta A$ is hermitian. Applying the preceding corollary, we have $A=0$ and so $B=0$, which shows $d \Phi$ is univalent.

q. e. d.

Since $d \Phi$ is univalent, there exists an analytic inverse map of $\Phi$ (p.80[1]), which we denote by $\Psi$. Thus we have

Proposition 2. Any complex regular matrix $\lambda$ can be written in one and only one way as the product $\lambda=\eta v$ of a positive definte hermitian matrix $\eta$ and a untary matrix $v$. The map

$$
\Psi: \mathrm{GL}(n, \boldsymbol{C}) \longrightarrow H^{+}(n) \times U(n)
$$

defined by this decompositıon gives an analytıc homeomorphism of $\mathrm{GL}(n, \boldsymbol{C})$ to $H^{+}(n) \times U(n)$ with respect to their usual analytic structures.

Remark. It easily follows from Propositions 1 and 2 that any $\lambda \in \mathrm{GL}(n, \boldsymbol{C})$ can also be decomposed analytically in one and only one way as $\lambda=u^{\prime} \eta^{\prime}$, where $v^{\prime} \in U(n)$ and $\eta^{\prime} \in H^{+}(n)$.

\section{$\S 3$. Existence Theorem.}

Let $(M, F, \hat{g})$ denote a complex manifold of odd complex dimension $2 m+1$ $(\geqq 3)$ with complex structure $F$ and hermitian metric $\hat{g}$ and let $M$ be covered by a system of coordinate neighborhoods $\mathcal{O}=\left\{O_{i}\right\}$. We shall suppose $M$ has a $\Omega$-structure. 
Since $\Omega_{\imath}$ is of $\operatorname{rank} 2 m$, if we set

$$
D_{\imath}(p)=\left\{X ; X \in T_{p}\left(O_{\imath}\right), \tau(X) \Omega_{i}=0\right\} \quad \text { for any } p \in O_{\imath},
$$

where $\tau(X) \Omega_{\imath}$ is defined by $\left(\tau(X) \Omega_{\imath}\right)(Y)=\Omega_{\imath}(X, Y)$ for any $Y \in T_{p}\left(O_{\imath}\right)$, then the correspondence $p \mapsto D_{i}(p)$ gives a distribution $D_{\imath}$ on each $O_{\imath}$ of complex dimension 1 and of class $C^{\infty}$. The condition $\Omega_{i}=f_{\imath j} \Omega_{\text {, on }} O_{\imath} \cap O_{j} \neq \phi$, where $f_{\imath \jmath}$ is a non-vanishing function, shows that $D_{i}=D_{\jmath}$ on $O_{i} \cap O_{\jmath}$, that is, $D_{2}$ is globally extended to a distribution $D$ on $M$ of complex dimension 1 and of class $C^{\infty}$.

On each $0_{i}$ we can choose a field of unitary frames $\left\{E_{i 1}, \cdots, E_{i 2 m}, N_{i}\right\}$ of class $C^{\infty}$ with respect to $\hat{g}$ such that the last vector $N_{\imath}$ belongs to the distribution $D$. The transformation of the field of such unitary frames on $O_{\imath} \cap O_{j} \neq \phi$ is of the form

$$
T_{\imath j}=\left(\begin{array}{cc|cc}
\tau_{\imath \jmath} & \vdots & & \\
& 0 & 0 & \\
0, \cdots 0, \tau^{\prime}{ }_{\imath \jmath} & & \\
& & & 0 \\
0 & & \bar{\tau}_{\imath \jmath} & \vdots \\
& & 0, \cdots 0, \bar{\tau}^{\prime}{ }_{\imath \jmath}
\end{array}\right)
$$

where $\tau_{\imath j} \in U(2 m), \tau^{\prime}{ }{ }_{j} \in U(1)$ and $\bar{\tau}_{\imath \jmath}$ denotes the complex conjugate of $\tau_{\imath \jmath}$.

The matrix $W_{\imath}$ consisting of components of the form $\Omega_{\imath}$ relative to the field of unitary frames defined on $0_{i}$ has the form

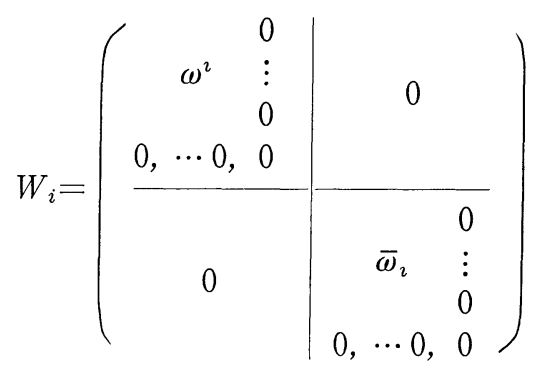

where $\omega_{i}$ is a regular $(2 m, 2 m)$-skew symmetric matrix. As a consequence of Proposition 2, $\omega_{i}$ is written as

$$
\omega_{i}=\xi_{i} \bar{\sigma}_{\imath}
$$

where $\xi_{i} \in H^{+}(2 m)$ and $\sigma_{i} \in U(2 m)$, and the components of $\xi_{\imath}$ and $\sigma_{\imath}$ depend analytically on those of $\omega_{i}$. So if we set 
ON THE EXISTENCE OF A COMPLEX A. C. STRUCTURE

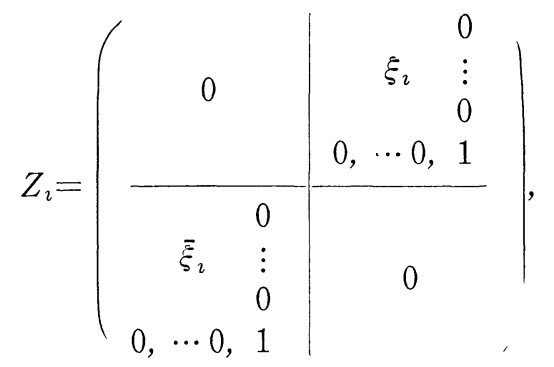

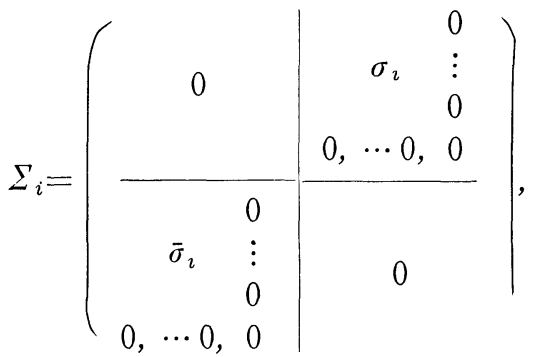

then (3.1) is equivalent to

$$
W_{\imath}=Z_{\imath} \Sigma_{\imath}
$$

Since $\omega_{i}$ is a skew symmetric matrix and $\sigma_{\imath} \in U(2 \mathrm{~m})$, we have

$$
\xi_{i} \bar{\sigma}_{i}=-{ }^{t} \bar{\sigma}_{\imath}{ }^{t} \xi_{i}=-\sigma_{\imath}^{-1} t \xi_{\imath}
$$

and thus

$$
\xi_{i}=-\sigma_{\imath}^{-1}{ }^{t} \xi_{i} \bar{\sigma}_{\imath}^{-1}=\sigma_{\imath}^{-1} t \xi_{\imath} \sigma_{i} \cdot\left(-\sigma_{\imath}^{-1} \bar{\sigma}_{i}^{-1}\right) .
$$

On the other hand it is easily checked

$$
\sigma_{\imath}^{-1} \xi_{\imath} \sigma_{i} \in H^{+}(2 m), \quad-\sigma_{\imath}^{-1} \bar{\sigma}_{\imath}^{-1} \in U(2 m) .
$$

Therefore by the uniqueness of the decomposition we have

$$
\xi_{i}=\sigma_{\imath}^{-1}{ }_{\imath}^{t} \xi_{\imath} \sigma_{\imath}, \text { i. e., } \quad \sigma_{i} \xi_{i}={ }^{t} \xi_{\imath} \sigma_{\imath}
$$

and

$$
\sigma_{\imath} \bar{\sigma}_{i}=-I_{2 m} .
$$

Where $I_{k}$ denote the unit $(k, k)$-matrix. The condition (3.3) is equivalent to 


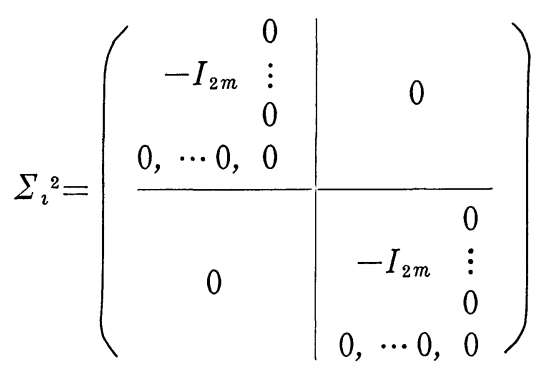

Next, the relation $\Omega_{i}=f_{\imath}, \Omega$, on $O_{\imath} \cap O_{j} \neq \phi$ is represented as

$$
W_{i}={ }^{t} T_{\imath \jmath} * f_{\imath \jmath}, T_{\imath \jmath}
$$

where

$$
* f_{\imath j}=\left(\begin{array}{c|c}
f_{\imath \jmath} I_{2 m+1} & 0 \\
\hline 0 & \bar{f}_{i \jmath} I_{2 m+1}
\end{array}\right)
$$

or equivalently as

$$
\omega_{i}={ }^{t} \tau_{\imath \jmath} f_{\imath j} \omega_{\jmath} \tau_{\imath \jmath}
$$

relative to the field of unitary frames defined on $O_{\imath}$. If we substitute (3.1) to this relation, we have

$$
\xi_{\imath} \bar{\sigma}_{i}={ }^{t} \tau_{\imath \jmath} f_{\imath j} \xi_{j} \bar{\sigma}_{\jmath} \tau_{\imath j}={ }^{t} \tau_{\imath j} \xi_{\jmath} \bar{\tau}_{\imath \jmath} \bar{\tau}_{i j}^{-1} f_{\imath \jmath} \bar{\sigma}_{\jmath} \tau_{\imath \jmath} .
$$

Since ${ }^{t} \tau_{\imath j} \xi_{j} \bar{\tau}_{\imath j} \in H^{+}(2 m)$ and $\bar{\tau}_{i j}^{-1} f_{\imath \jmath} \bar{\sigma}_{\jmath} \tau_{\imath j} \in U(2 m)$, by the uniqueness of the decomposition of this type, we have

$$
\xi_{i}={ }^{t} \tau_{\imath j} \xi_{\jmath} \bar{\tau}_{\imath \jmath}, \quad \sigma_{i}=\tau_{i j}^{-1} \bar{f}_{\imath j} \sigma, \bar{\tau}_{\imath \jmath},
$$

or

$$
Z_{i}={ }^{t} T_{\imath \jmath} Z_{\jmath} T_{\imath \jmath}, \quad \Sigma_{i}=T_{i j}^{-1} * \bar{f}_{\imath \jmath} \Sigma_{\jmath} T_{\imath \jmath} .
$$

Thus, there is a global $(0,2)$-tensor field $g$ of class $C^{\infty}$ on $M$ defined by the set $\left\{Z_{\imath} ; O_{i} \in \mathcal{O}\right\}$. (See [8].) Since each $\xi_{\imath}$ is positive definite, $g$ is regarded as a positive definite hermitian metric on $M$. Also there is a local $(1,1)$-tensor field $G_{i}$ of class $C^{\infty}$ and of rank $2 m$ defined by $\Sigma_{\imath}$ on each $O_{\imath}$ in such a way that the condition $G_{i}=\bar{f}_{\imath j} G_{j}$ is satisfied on $O_{\imath} \cap O_{j} \neq \phi$.

Now, we shall define on each $O_{\imath}$ vector fields $U_{\imath}$ and $V_{\imath}, 1$-forms $u_{\imath}$ and $v_{i}$ and $(1,1)$-tensor field $H_{\imath}$ respectively by 
ON THE EXISTENCE OF A COMPLEX A. C. STRUCTURE

$$
\begin{aligned}
& U_{i}=N_{\imath}, \\
& V_{i}=-F N_{\imath}, \\
& u_{i}=\iota\left(U_{\imath}\right) g, \\
& v_{i}=\imath\left(V_{\imath}\right) g, \\
& H_{i}=-F G_{i} .
\end{aligned}
$$

Then from (3.2), (3.3)', and (3.5) the following relations are easily varified:

$$
\begin{gathered}
F U_{i}=-V_{\imath}, \quad F V_{i}=U_{\imath}, \\
g\left(U_{\imath}, U_{\imath}\right)=g\left(V_{\imath}, V_{\imath}\right)=1, \quad g\left(U_{\imath}, V_{\imath}\right)=0, \\
G_{i} F=-F G_{i}=H_{\imath}, \quad H_{\imath} F=-F H_{i}=-G_{i}, \\
G_{i} U_{i}=G_{i} V_{i}=H_{\imath} U_{i}=H_{\imath} V_{\imath}=0, \\
v_{i} \circ G_{i}=v_{i} \circ G_{i}=u_{i} \circ H_{i}=v_{i} \circ H_{i}=0, \\
G_{i}{ }^{2}=H_{\imath}{ }^{2}=-I+u_{\imath} \otimes U_{i}+v_{\imath} \otimes V_{\imath}, \\
H_{\imath} G_{i}=-G_{i} H_{i}=F+u_{i} \otimes V_{i}-v_{i} \otimes U_{\imath} .
\end{gathered}
$$

Furthermore, taking account of $\tau_{\imath j} \in U(2 m), \tau^{\prime}{ }_{\imath j} \in U(1)$ and $f_{\imath j} \in U(1)$ on $O_{\imath} \cap O_{\jmath}$ $\neq \phi$, and by (3.4), we obtain

$$
\left\{\begin{array} { l } 
{ u _ { i } = a u _ { j } + b v _ { \jmath } , } \\
{ v _ { i } = - b u _ { \jmath } + a v _ { \jmath } , }
\end{array} \quad \left\{\begin{array}{l}
G_{i}=c G_{\jmath}+d H_{\jmath}, \\
H_{i}=-d G_{j}+c H_{\jmath} .
\end{array}\right.\right.
$$

where $a, b, c$, and $d$ are real valued functions satisfying $a^{2}+b^{2}=1$, and $c^{2}+d^{2}=1$.

The preceding argument shows that the set $\left\{\left(O_{\imath}, u_{\imath}, v_{\imath}, G_{i}, H_{\imath}\right): O_{i} \in \mathcal{O}\right\}$ defines a complex almost contact structure on $M$. Thus we have

THEOREM. Let $(M, F, \hat{g})$ denote a complex manifold of odd complex dimension $2 m+1(\geqq 3)$ with complex structure $F$ and hermitian metric $\hat{g}$. If $M$ has a $\Omega$-structure, then there are a hermitian metric $g$ and a complex almost contact structure on $M$.

Remark. The significance of the Theorem proved in the preceding way is that if we suppose the original hermitian metric $\hat{g}$ and 2 -forms $\Omega_{i}$ 's are of class $C^{\omega}$, then we get a hermitian metric $g$ and $(1,1)$-tensor fields $G_{i}$ 's of class $C^{\omega}$.

It might be convenient to have a metric $\tilde{g}$ satisfying $\tilde{g}\left(G_{i} X, Y\right)=-\tilde{g}\left(X, G_{i} Y\right)$ and $\tilde{g}\left(H_{\imath} X, Y\right)=-\tilde{g}\left(X, H_{\imath} Y\right)$ on each $O_{\imath}$. We get such a metric from $g$ in the Theorem in the following way.

First we define $\tilde{g}_{\imath}$ on each $O_{\imath}$ by

$$
\tilde{g}_{\imath}(X, Y)=g(X, Y)+\frac{1}{2}\left\{g\left(G_{\imath} X, G_{\imath} Y\right)+g\left(H_{\imath} X, H_{\imath} Y\right)\right\} .
$$


Then by (3.7) we see $\tilde{g}_{\imath}(X, Y)=\tilde{g}_{j}(X, Y)$ on $O_{\imath} \cap O_{j} \neq \phi$. This shows that $\tilde{g}_{\imath}$ is extended to a global metric, which we denote by $\tilde{g}$. It is easily checked that $\tilde{g}$ satisfies the required conditions on each $O_{\imath}$, namely, $\tilde{g}\left(G_{i} X, Y\right)=-\tilde{g}\left(X, G_{i} Y\right)$ and $\tilde{g}\left(H_{\imath} X, Y\right)=-\tilde{g}\left(X, H_{\imath} Y\right)$ as well as $\tilde{g}(F X, Y)=-\tilde{g}(X, F Y)$. However changing metrics of this type does not affect the definitions of $U_{\imath}, V_{\imath}, u_{\imath}$, and $v_{i}$. In fact, this is easily checked by (3.5) and (3.6).

Remark. By slightly modifying the argument in this section we have the following :

Instead of a $\Omega$-structure, if there is on each $O_{\imath}$ a 2 -form $\Omega_{\imath}$ of type $(2,0)$ satisfying $\left(\Omega_{\imath}\right)^{n} \neq 0(n \leqq m)$ and $\Omega_{i}=f_{\imath \jmath} \Omega_{\jmath}, f_{\imath j} \in U(1)$ on $O_{\imath} \cap O_{j} \neq \phi$, then there is a hermitian metric $g$ on $M$ such that the non-zero eigenvalues of each $\Omega_{\imath}$ with respect to $g$ are only $\imath$ and $-\imath$. (See $(3.3)^{\prime}$.)

\section{BIBLIOGRAPHY}

[1] C. Chevalley, Theory of Lie groups I, Princeton Univ. Press, 1946.

[2] D. E. Blair, S. Ishihara and G. D. Ludden, Projectable Almost Complex Contact Structure, to appear.

[3] W.M. Boothiy, Homogeneous Complex Contact Manifolds, Proc. Symp. Pure Math., Vol. III, 144-154, Amer. Math. Soc., 1961.

[4] Y. Hatakeyama, On the existence of Riemannian metrics associated with 2-form of rank 2r, Tōhoku Math. J., 14 (1962), 162-166.

[5] S. Ishinara and M. Konishi, Complex Almost Contact Structures and Fiberings, to appear.

[6] S. Ishinara and M. Konishi, Complex Almost Contact Manifolds, to appear.

[7] S. Kobayashi, Remarks on Complex Contact Manifolds, Proc. Amer. Math. Soc., 10 (1959), 164-167.

[8] K. YANo, Differential Geometry on Complex and Almost Complex Spaces, Pergamon Press, 1965.

Tokyo Institute of TECHNOLOGy 